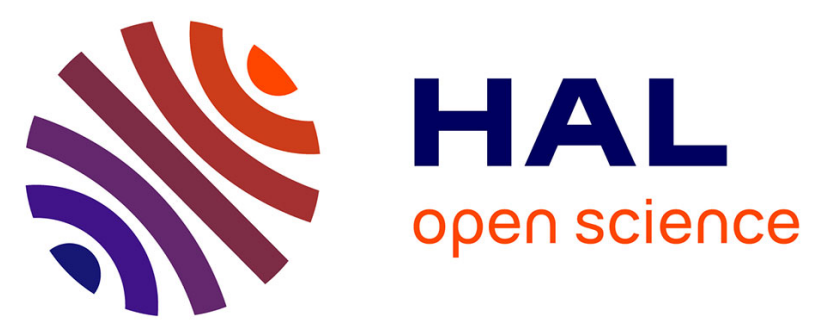

\title{
Columnar structure formation of a dilute suspension of settling spherical particles in a quiescent fluid
}

Sander G. Huisman, Thomas Barois, Mickaël Bourgoin, Agathe Chouippe,

Todor Doychev, Peter Huck, Carla E. Bello Morales, Markus Uhlmann, Romain Volk

\section{To cite this version:}

Sander G. Huisman, Thomas Barois, Mickaël Bourgoin, Agathe Chouippe, Todor Doychev, et al.. Columnar structure formation of a dilute suspension of settling spherical particles in a quiescent fluid. Physical Review Fluids, 2016, 1 (7), pp.74204. 10.1103/PhysRevFluids.1.074204 . hal-01565116

\author{
HAL Id: hal-01565116 \\ https://hal.science/hal-01565116
}

Submitted on 29 Sep 2017

HAL is a multi-disciplinary open access archive for the deposit and dissemination of scientific research documents, whether they are published or not. The documents may come from teaching and research institutions in France or abroad, or from public or private research centers.
L'archive ouverte pluridisciplinaire HAL, est destinée au dépôt et à la diffusion de documents scientifiques de niveau recherche, publiés ou non, émanant des établissements d'enseignement et de recherche français ou étrangers, des laboratoires publics ou privés. 


\title{
Columnar structure formation of a dilute suspension of settling spherical particles in a quiescent fluid
}

\author{
Sander G. Huisman, ${ }^{1}$ Thomas Barois, ${ }^{1}$ Mickaël Bourgoin, ${ }^{1}$ Agathe Chouippe,${ }^{2}$ Todor Doychev, ${ }^{2}$ \\ Peter Huck, ${ }^{1}$ Carla E. Bello Morales, ${ }^{1,3}$ Markus Uhlmann, ${ }^{2}$ and Romain Volk ${ }^{1}$ \\ ${ }^{1}$ Université Lyon, Ens de Lyon, Université Claude Bernard, CNRS, Laboratoire de Physique, \\ F-69342 Lyon, France \\ ${ }^{2}$ Institute for Hydromechanics, Karlsruhe Institute of Technology, 76131 Karlsruhe, Germany \\ ${ }^{3}$ CICATA-Qro, Instituto Politécnico Nacional, 76090 Querétaro, Mexico
}

(Received 22 June 2016; published 11 November 2016)

\begin{abstract}
The settling of heavy spherical particles in a column of quiescent fluid is investigated. The performed experiments cover a range of Galileo numbers $(110 \leqslant \mathrm{Ga} \leqslant 310)$ for a fixed density ratio of $\Gamma=\rho_{p} / \rho_{f}=2.5$. In this regime the particles are known to show a variety of motions [Jenny, Dušek, and Bouchet, Instabilities and transition of a sphere falling or ascending freely in a Newtonian fluid, J. Fluid Mech. 508, 201 (2004)]. It is known that the wake undergoes several transitions for increasing Ga resulting in particle motions that are successively vertical, oblique, oblique oscillating, and finally chaotic. Not only does this change the trajectory of single, isolated, settling particles, but it also changes the dynamics of a swarm of particles as collective effects become important even for dilute suspensions with volume fraction up to $\Phi_{V}=O\left(10^{-3}\right)$, which are investigated in this work. Multicamera recordings of settling particles are recorded and tracked over time in three dimensions. A variety of analyses are performed and show a strong clustering behavior. The distribution of the cell areas of the Voronoï tessellation in the horizontal plane is compared to that of a random distribution of particles and shows clear clustering. Moreover, a negative correlation was found between the Voronoï area and the particle velocity; clustered particles fall faster. In addition, the angle between adjacent particles and the vertical is calculated and compared to a homogeneous distribution of particles, clear evidence of vertical alignment of particles is found. The experimental findings are compared to simulations.
\end{abstract}

DOI: 10.1103/PhysRevFluids.1.074204

\section{INTRODUCTION}

The settling of a sphere in a quiescent viscous fluid is a long-standing problem, already explored by Newton [1] in the 17th century. The problem is simple only in appearance. The usual picture of a straight settling trajectory with a constant terminal velocity resulting from the balance between buoyancy and viscous drag is an important but only marginal situation in a much richer landscape of possible settling regimes.

A sufficiently small or slowly settling sphere (i.e., in a low Reynolds number approximation, so that the flow around the particle can be approximated as a Stokes flow) with diameter $d$ and density ratio $\Gamma=\rho_{p} / \rho_{f}$ (where $\rho_{p}$ is the particle density and $\rho_{f}$ the density of the fluid) surrounded by a fluid with viscosity $v$ will indeed settle along a straight vertical path, reaching a steady terminal vertical velocity $V$, where the linear viscous drag (due to the Stokes flow around the particle) $F_{D}=\frac{1}{8} C_{D}\left(\operatorname{Re}_{p}\right) \rho_{f} \pi d^{2} v_{s}^{2}$ [with $\operatorname{Re}_{p}=d V / v$ the particulate Reynolds number and $C_{D}\left(\operatorname{Re}_{p}\right)$ the drag coefficient, which is simply $24 / \operatorname{Re}_{p}$ for a sphere in the limit $\operatorname{Re}_{p} \ll 1$ ] is balanced by the buoyancy force, so that $V=(\Gamma-1) g d^{2} /(18 v)$. Several successive scenarios arise as the size or density of the particle increases and the particulate Reynolds number increases.

\section{A. Finite size effects}

At finite particulate Reynolds number, the flow around a sphere departs from a simple Stokes flow and eventually develops wake instabilities. Such instabilities have been extensively studied 


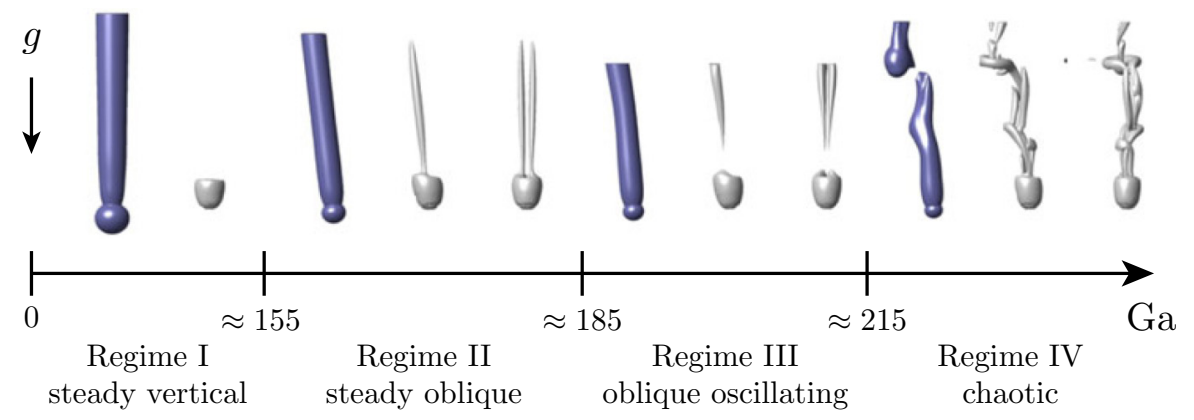

FIG. 1. Four regimes of single particle settling in ambient fluid as a function of Ga for $\Gamma=1.5$. The snapshots are for (from left to right) $\mathrm{Ga}=144,178,190$, and 250. The visualizations are taken from Ref. [9] and show (in purple) the isocontour for which the velocity is $1.2 V_{g}$ and show (in gray) from multiple angles the isocontour of a $\lambda_{2}$ criterion.

(numerically and experimentally) in the past 15 years for the case of fixed spheres in a flow [2-5]. The global picture is now clear, with several successive instabilities taking place as $\mathrm{Re}_{p}$ increases: from a steady axisymmetric wake at low $\mathrm{Re}_{p}$ to, first, a wake with steady planar double-threaded vortices (above $\operatorname{Re}_{p} \simeq 210$ ), then an unsteady time-periodic vortex shedding wake (above $\operatorname{Re}_{p} \simeq 275$ ), and finally a transition towards a fully three dimensional chaotic wake (above $\operatorname{Re}_{p} \simeq 360$ ). These successive modifications of the wake have two main implications. First, the value of the drag coefficient $C_{D}$ departs from the simple $24 / \operatorname{Re}_{p}$ law as $\mathrm{Re}_{p}$ increases, reaching eventually a full nonlinear drag regime (with an almost constant value of $C_{D}$ at very high values of $\operatorname{Re}_{p}$ when the wake becomes fully turbulent).

Second, in the case of a free-falling sphere (with additional degrees of freedom compared to the case of a fixed sphere) these wake instabilities induce particle path instabilities, marking a departure from a simple vertical trajectory. The settling regime of a free-falling sphere is controlled by two dimensionless parameters: the density ratio $\Gamma=\rho_{p} / \rho_{f}$ and the Galileo number Ga $=V_{g} d / \nu$, which can be thought of as a Reynolds number based on the gravitational velocity $V_{g}=[(\Gamma-1) g d]^{1 / 2}$. A sphere settling in finite Ga regimes (hence beyond the Stokes approximation) is referred to as a "finite size sphere" (by definition the settling problem concerns the case $\Gamma>1$ ). The settling Reynolds number $\operatorname{Re}_{p}=V d / v$ is then an output parameter of the problem. The path instabilities at finite values of Ga are now well characterized and have been deeply investigated numerically [6] and experimentally $[7,8]$ in the past decade for single finite size free-falling spheres; $\mathrm{cf}$. the visualizations in Fig. 1 (see also the review by Ern et al. [10]).

\section{B. Collective effects}

The case of many particles settling simultaneously raises further complexity, as long-range multiparticle hydrodynamic interactions emerge. In the limit of point particles (or equivalently in the limit $\mathrm{Ga} \ll 1$ ), the collective settling of such an ensemble of spheres and the underlying hydrodynamic interactions can be efficiently studied using Stokesian dynamics methods [11,12]. This approach yields a satisfactory quantitative comparison with experiments [13], although several questions remain, in particular, regarding the induced fluctuations and the correlation lengths of particles and flow motion. Much less is known, however, for the case of a settling ensemble of finite size particles. Systematic experimental studies remain scarce. Parthasarathy and Faeth [14] and Mizukami et al. [15] performed a series of experiments in dilute conditions (volume fraction of particles $\Phi_{v}<1 \times 10^{-4}$ ) in a range of Ga from 40 to 340 . Their experiments focused on measuring the fluctuations of the flow induced by the interacting wakes of the particles and showed that in dilute regimes a linear superposition of wakes gives a good approximation. It is only recently that accurate numerical simulations of a large number of fully resolved finite size particles settling collectively 
and fully coupled with the fluid have become possible [16,17], mainly thanks to immersed boundary methods combined with direct numerical simulations of the Navier-Stokes equation. These methods permit a description of the coupling between the particle and the surrounding flow at the interface level. Uhlmann and Doychev $[9,18]$ reported recently using this method that, depending on the value of $\mathrm{Ga}$, particles may tend (or not) to align in columnar clusters along their wakes, resulting in an enhancement of their settling velocity (the average settling velocity was found up to $12 \%$ faster than for individual particles). Such a clustering and settling enhancement was observed for $\mathrm{Ga}=178$ (hence in a regime of steady oblique motion for individual settling particles; see Fig. 1), but was not observed for $\mathrm{Ga}=121$ (when individual particles settle along a steady vertical path). This study shows that the interplay of individual wake instabilities and collective interactions is crucial to understand the settling of an ensemble of finite size spheres, even in relatively dilute conditions (in their study $\Phi_{v}=5 \times 10^{-3}$ ). However, the computational cost of these simulations does not yet allow a systematic exploration of the parameter space (density ratio, Galileo number, and volume fraction). At the moment these studies [9,18] only comprise three parameter points at a single value of the density ratio and for two different solid volume fractions and Galileo numbers. We propose here to explore the collective settling of finite size spheres experimentally, with the goal to broaden the range of parameters, in particular regarding the role of the Galileo number around the first wake instabilities. In the present article we therefore address the experimental counterpart of the aforementioned simulations, by exploring the settling behavior of a swarm of dense finite size particles. We look more particularly at the eventual emergence of clustering and columnar alignment, and its impact on local and global settling velocity, for increasing values of the Galileo number in the range $\mathrm{Ga} \in[110,310]$ and comparable seeding densities of the order of $\Phi_{V} \approx 5 \times 10^{-4}$. We compare our experimental data with an existing numerical simulation [18] with $\mathrm{Ga}=178$, in the steady oblique regime when considering an isolated particle, and at a solid volume fraction $\Phi_{V}=4.8 \times 10^{-4}$ of the same order in the experiments. The numerical method, the grid resolution, and the configuration are identical to case M178 of Uhlmann and Doychev [9]. The solid volume fraction, however, was reduced by a factor of 10: it employs the immersed boundary method of Uhlmann [16] on a triply periodic box elongated in the direction of gravity with a treatment of collisions with a repulsive force [19]. The simulation domain has an extension of 85 particle diameters in the horizontal directions and 171 diameters in the vertical direction with a uniform grid resolution $\Delta x$ such that $d / \Delta x=24$. This simulation covers 1756 gravitational time units.

\section{EXPERIMENTAL DETAILS}

In the current work we look at the collective effects of heavy spherical particles settling in a column of quiescent liquid; see Figs. 2(c) and 2(d). The $2 \mathrm{~m}$ high column has a square cross section with sides of $0.3 \mathrm{~m}$. In order to vary Ga two sizes of glass particles $(d=2 \mathrm{~mm}$ and $d=3 \mathrm{~mm})$ with density $\rho_{p}=2.5 \times 10^{3} \mathrm{~kg} / \mathrm{m}^{3}$ and two fluid viscosities $\left(v=2.05 \times 10^{-6} \mathrm{~m}^{2} / \mathrm{s}\right.$ and $v=3.15 \times 10^{-6} \mathrm{~m}^{2} / \mathrm{s}$ created using a mixture of water and $\mathrm{Ucon}^{\mathrm{TM}}$ oil) are used, resulting in $\mathrm{Ga} \in[110,310]$. Constant seeding of heavy particles is accomplished by gradually pouring particles on a stack of six perforated meshes, which has become the de facto standard; see, e.g., Ref. [14]. The particles that leave the last mesh were checked to have a Voronoï area distribution closely resembling a random set of Poisson points (RPP) distribution (and very far from the Voronoï distribution farther downstream) by injecting particles in a very shallow tank and then taking photographs of the injected particles. The columns are therefore not introduced because of the mesh but are formed during descent. The particles travel roughly $1 \mathrm{~m}$ before entering the field of view of the cameras, attaining their terminal velocity far before entering the view. For our largest particles this distance of $1 \mathrm{~m}$ corresponds to roughly $330 \mathrm{~d}$. Numerical simulations [9] suggest that at least a distance of $250 \mathrm{~d}$ is needed in order to observe clusters and columns [9]. The falling particles are recorded by a set of three Flare 2M360-CL cameras from IO Industries at up to 240 frames per second at a resolution of $2048 \times 1088$ using an $8 \mathrm{~mm}$ focal length lens covering a height of roughly $0.6 \mathrm{~m}$. The particles are coated black and backlight illumination is employed for enhanced contrast. The cameras are calibrated using an 

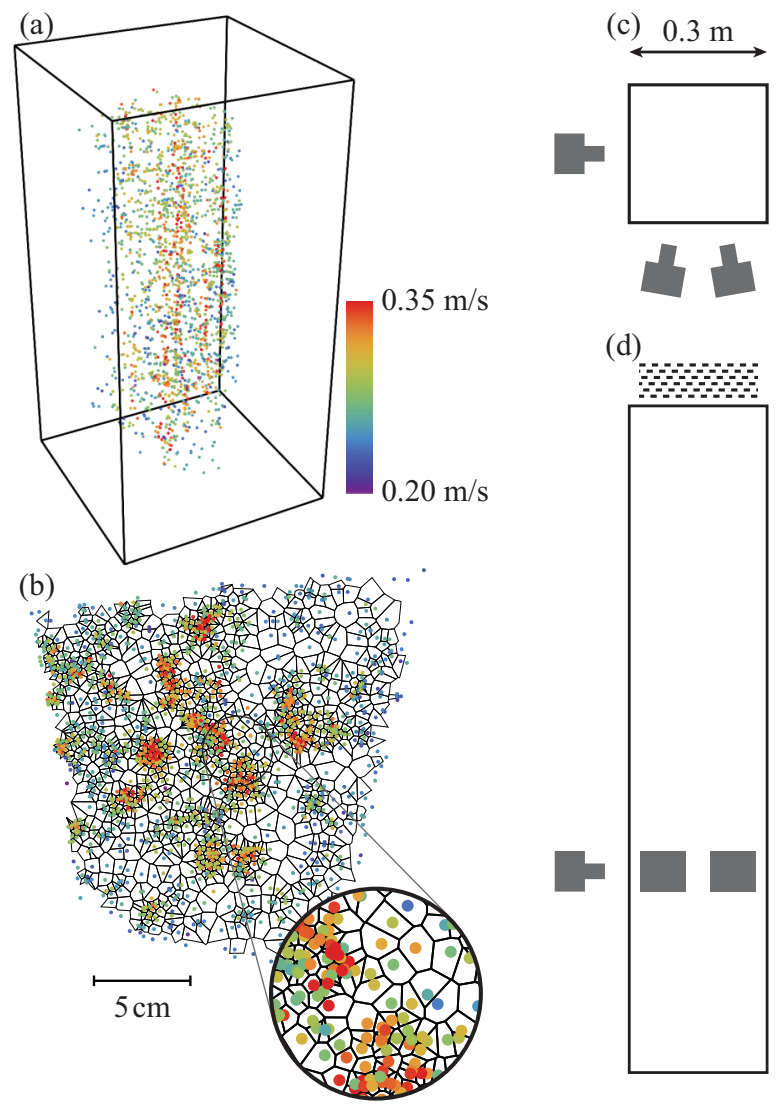

(d)

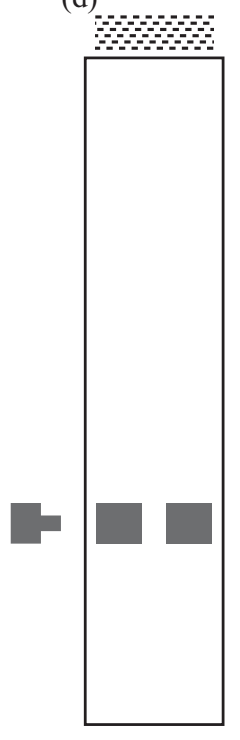

FIG. 2. (a) Three-dimensional reconstructing of settling particles with $\mathrm{Ga}=170$ colored by their velocities. Short sides of the box are $0.3 \mathrm{~m}$. (b) Top view of (a) including scale bar and the corresponding Voronoï tessellation; see also the detailed $(3 \times)$ zoom. (c) Top view with the camera arrangement. (d) Side view with the injector visible at the top. The injector comprises stacked perforated metal meshes in order to randomly inject the particles.

in situ calibration method [20] achieving a subradius resolution of roughly $300 \mu \mathrm{m}$. Picked from a set of experiments are those for which the volume fractions $\Phi_{V}$ are constant and comparable to one another (see Table I). Note that the injection is done manually and the volume concentration is only known a posteriori. Injections that are too short do not form columns and only show transient behavior and are therefore rejected. Furthermore, we look for experiments where the number of particles in the field of view remains roughly constant. The selected experiments all have a constant volume fraction in the range $1 \times 10^{-4}-1 \times 10^{-3}$ and have sufficiently long duration of injection such that columns can potentially be formed. In particular, we make sure to omit the transient part of each experiment, at the beginning when particles start entering the measurement volume and at the end when they leave it. These experiments have the following Galileo numbers: $\mathrm{Ga}=110(2)$, $\mathrm{Ga}=170(2), \mathrm{Ga}=200(2)$, and $\mathrm{Ga}=310(3)$ where the values in parentheses are the number of experiments; see also Table I. Note that the values for Ga are rounded to the nearest 10 to reflect the errors in $\rho_{f}, d$, and $v$, which leads to an estimated error of 10 for the Galileo number. In the recorded imagery, particles are detected and using standard particle tracking velocimetry (PTV) the locations of the particles are tracked over time and three-dimensional space. A snapshot of the reconstructed particle positions can be seen in Fig. 2(a) for the case $\mathrm{Ga}=170$. Visual inspection reveals already clear vertical trails of particles. 
TABLE I. Overview of the experimental parameters. $\operatorname{Re}_{\infty}$ is defined as $\operatorname{Re}_{\infty}=V_{\infty} d / v$ and $V_{g}=$ $[(\Gamma-1) g d]^{1 / 2}$.

\begin{tabular}{cccccccccc}
\hline \hline $\mathrm{Ga}$ & $\Gamma$ & $d(\mathrm{~mm})$ & $v\left(10^{-6} \mathrm{~m}^{2} / \mathrm{s}\right)$ & $V_{g}(\mathrm{~m} / \mathrm{s})$ & $V_{\infty}(\mathrm{m} / \mathrm{s})$ & $\langle V\rangle(\mathrm{m} / \mathrm{s})$ & $\langle V\rangle / V_{\infty}$ & $\operatorname{Re}_{\infty}$ & $\phi_{V}\left(10^{-5}\right)$ \\
\hline 110 & 2.5 & 2.0 & 3.15 & 0.17 & 0.18 & 0.22 & 1.19 & 120 & 38 \\
110 & 2.5 & 2.0 & 3.15 & 0.17 & 0.18 & 0.21 & 1.13 & 120 & 20 \\
170 & 2.5 & 2.0 & 2.05 & 0.17 & 0.25 & 0.31 & 1.25 & 240 & 49 \\
170 & 2.5 & 2.0 & 2.05 & 0.17 & 0.25 & 0.30 & 1.21 & 240 & 56 \\
200 & 2.5 & 3.0 & 3.15 & 0.21 & 0.30 & 0.36 & 1.17 & 290 & 79 \\
200 & 2.5 & 3.0 & 3.15 & 0.21 & 0.30 & 0.38 & 1.23 & 290 & 100 \\
310 & 2.5 & 3.0 & 2.05 & 0.21 & 0.33 & 0.37 & 1.10 & 490 & 84 \\
310 & 2.5 & 3.0 & 2.05 & 0.21 & 0.33 & 0.39 & 1.16 & 490 & 79 \\
310 & 2.5 & 3.0 & 2.05 & 0.21 & 0.33 & 0.39 & 1.17 & 490 & 76 \\
\hline \hline
\end{tabular}

\section{RESULTS}

The velocity of the particles is inferred from the particle trajectories and is defined as positive in the direction of gravity; see also Fig. 2(a). Visually we already observe the formation of high-speed (orange-red) columns inside the measurement volume. Moreover, during the experiment one can clearly see the column extend way beyond the measurement section. We estimate that the columns are at least $1.5 \mathrm{~m}$ long without any sign that they are unstable or that they break up. The probability density function (PDF) of the velocity as a function of Ga can be found in Fig. 3(a). The mean settling velocities of isolated particles are included as colored dashed lines and increases with Ga. We complement our experimental data with numerical simulations [18] with comparable $\mathrm{Ga}=178$, $\Gamma=1.5$, and $\Phi_{V}=0.00048$; see Fig. 3(a). A large spread of the velocity above its isolated velocity is found, which supports the idea of an enhanced settling velocity in a suspension of particles. Figure 3(b) shows the velocities normalized by their isolated settling velocity. We note that the largest velocity enhancement is seen for $\mathrm{Ga}=170$. The speed enhancement for $\mathrm{Ga}=310$ is notably less than for the $\mathrm{Ga}=170$ and $\mathrm{Ga}=200$ cases; see also Table I. Several possible reasons might explain this behavior. First, the most probable explanation is that the chaotic wakes of the $\mathrm{Ga}=310$ particles might prohibit the formation of a stable train of settling particles, which is not the case for intermediate Ga. Second, the volume fraction necessary for "equivalent" clustering might be a
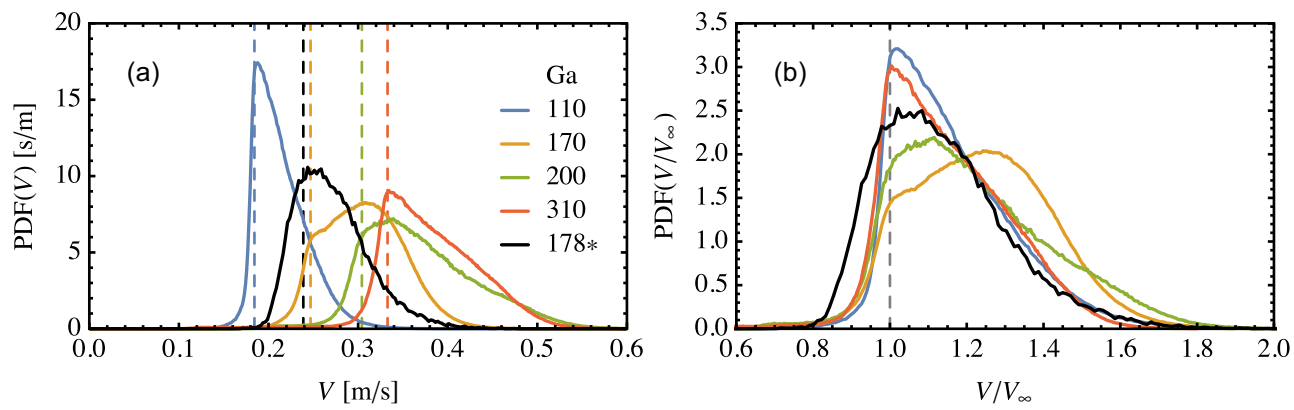

FIG. 3. (a) Velocity probability density function as a function of Ga. Multiple experiments for the same Ga are in agreement and are combined to improve the quality of the statistics. Dashed lines indicate the settling velocity of isolated particles $V_{\infty}$ for each Ga and are colored analogously. Starred data are from numerical simulations [18]; the data are made dimensional using the diameter and viscosity from the experimental $\mathrm{Ga}=170$ case. Note that $\Gamma=1.5$ for the numerical data $[9,18]$, while the experiments have $\Gamma=2.5$. (b) Vertical velocity normalized by the settling velocity of an isolated particle; see Table I. Same colors as in (a). Average velocities for each experiment can be found in Table I. 

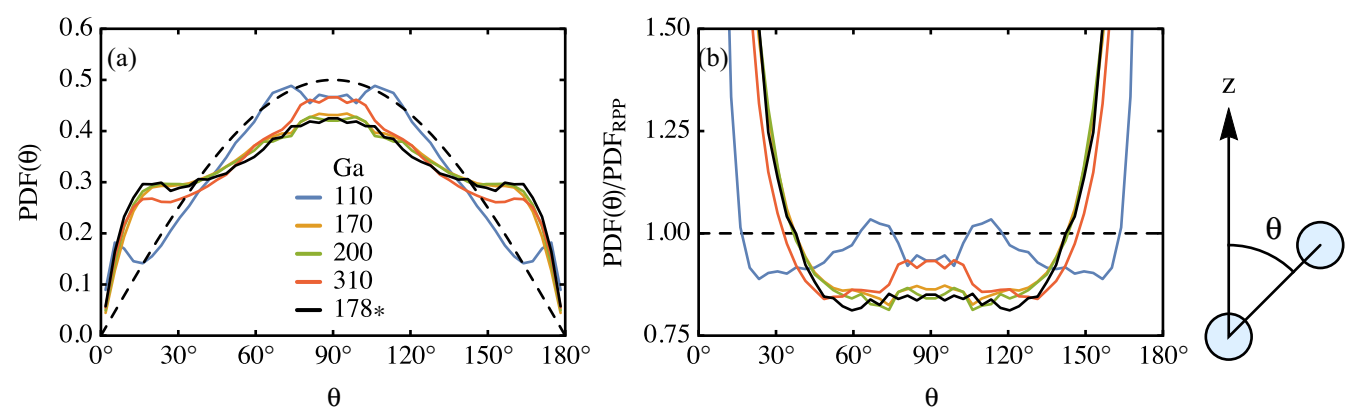

FIG. 4. (a) Angular pair probability density function for various Ga. The angle $\theta$ is defined as the angle between two adjacent particles and the vertical, as seen on the right. The dashed lines indicate the PDF for a set of Poisson particles (RPP), i.e., noninteracting particles that are placed randomly, and is given by $\mathrm{PDF}_{\mathrm{RPP}}=\sin (\theta) / 2$ and is shown for reference. The graph is, by definition, symmetric around $\theta=90^{\circ}$. (b) The data of (a) normalized by PDF $_{\mathrm{RPP}}$. Same colors as in (a). Data bigger than 1 are more likely to occur, while values smaller than 1 are less likely to occur as compared to RPP. Strong enhancement can be seen around $\theta=0^{\circ}$ and $\theta=180^{\circ}$.

function of Ga. The velocities lower than the mean isolated velocity [below 1 in Fig. 3(b)] are caused by the combined effects of nonmonodispersity, minute density differences, lack of roundness, and the unavoidable adherence of microbubbles. In addition, it is clear that particles drag fluid downwards with them, and, therefore, when fluid is going downward, an equal amount has to go up as per the continuity equation. This upward flow could cause isolated particles (particles in low density regions) to appear to have a lower velocity in the frame of reference of the laboratory. This effect cannot be disentangled by us as we do not have access to the velocity of the fluid. The experiments are performed in the center of a square box and any upward flow can thus go "around" the falling particles en masse; however, in the simulation there is no such possibility as the simulation domain is performed in a three-dimensional periodic box. In the simulation we therefore expect more particles with a velocity below $V / V_{\infty}=1$. Indeed, this can clearly be seen in Fig. 3(b). The strong enhancement of the velocity can be attributed to particles entering the wake of upstream (leading) particles, similar to what happens with aligned bubbles rising in tandem [21]. We therefore expect that particles tend to align vertically such that a collection of vertically clustered particles can fall faster as they would individually.

To investigate this vertical alignment hypothesis we look at the angle $\theta$ of a particle with other particles in its vicinity and the vertical; see the sketch in Fig. 4. The focus is at close-range interactions, and therefore the distance between two neighboring particles is limited to $8 d$. We plot the PDF of the angle $\theta$ as a function of Ga; see Fig. 4(a).

To compare our data we introduce the concept of a random set of Poisson point (RPP) particles. These noninteracting particles are independently and randomly placed inside a volume of choice. We use this as a reference and show that our particles (despite the low Re and relatively low $\Phi_{V}$ ) do interact with each other as their statistics strongly differ from that of RPP particles. For such a random set of points the distribution of the angle $\theta$ can be theoretically calculated and follows: $\mathrm{PDF}_{\mathrm{RPP}}=\sin (\theta) / 2$, where $\theta \in[0, \pi]$, following from the Jacobian of spherical coordinates. Each pair is considered twice and with a different angle $\theta$. The sum of these two angles is, however, $180^{\circ}$, which ensures that the PDF is indeed symmetric around $\theta=90^{\circ}$. For the low Ga case of $\mathrm{Ga}=110$ we see that the PDF [Fig. 4(a)] closely resembles the one that is found if one were to take random particles. We do find a slight increase around $\theta=0^{\circ}$ and a slight decrease around $\theta=90^{\circ}$ which might be caused by the mild clustering that we see from visual inspection, and which we show later using Voronoï analysis. For the high Ga cases ( $\mathrm{Ga} \geqslant 170)$, we see a very different behavior; namely, for $45^{\circ}<\theta<135^{\circ}$ we see a clear reduction in the number of neighbors [see also Fig. 4(b), where we present the distribution normalized by the RPP distribution]. So the chance of finding particles 

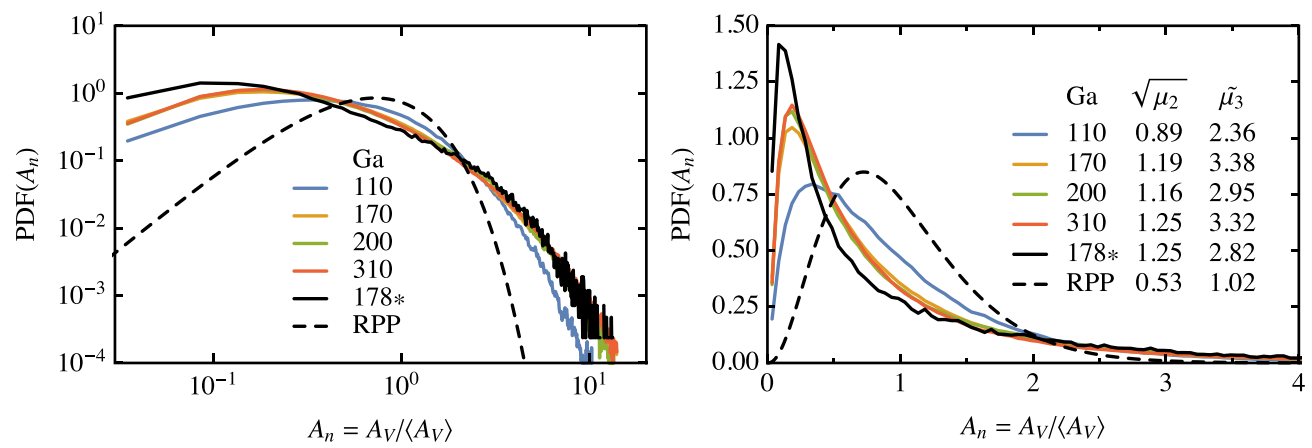

FIG. 5. Probability distribution of the normalized Voronoï areas in the horizontal plane. The corresponding probability density function for randomly distributed points (RPP) is shown as a dashed line. Left: Logarithmic axes. Right: Linear axes; the legend includes the standard deviation $\left(\sqrt{\mu_{2}}\right)$ and the skewness $\left(\tilde{\mu_{3}}\right)$ of $A_{n}$.

next to each other is significantly reduced for the high Ga cases. As a consequence, or rather a cause, particles are found to align vertically far more than a random set of Poisson particles; the PDF is found to be much higher for $\theta<45^{\circ}$ (or equivalently $\theta>135^{\circ}$ ), which can be more clearly seen in Fig. 4(b). Figure 4 has the inclusion of numerical data [18] with $\mathrm{Ga}=178, \Phi_{V}=0.00048$, and $\rho_{p} / \rho_{f}=1.5$, which is comparable to our set of parameters. For these data we find that angle-pair distribution is remarkably similar to the experimentally found distributions for $\mathrm{Ga}=170$, despite having a fairly different density ratio $\Gamma=\rho_{p} / \rho_{f}$. Indeed a difference in $\Gamma$ can have a profound impact on the stability of the wake as found by Refs. [6,7], though the influence is expected to be relatively small in the range $1.5<\Gamma<2.5$ for this value of the Galileo number. Note, however, that the phase spaces presented in Refs. [6,7] are for the case of vanishing $\Phi_{V}$, and these lines might shift significantly for increasing $\Phi_{V}$ as neighboring particles can trigger wake instabilities.

A look from the top further corroborates the hypothesis that these particles cluster and thereby form a rapidly settling group (or cluster) of particles; see Fig. 2(b). This top view shows the horizontal position of the particles colored by their velocities. Not only do we observe a set of high-density and low-density regions - far different from a RPP_-but also that the settling velocities are higher in high-density regions, which can be attributed to vertical alignment and further corroborates our view of the mechanism of enhanced settling velocity. The observation of relatively low- and high-density regions - different from RPP — can be substantiated by computing the Voronoi tessellation in the horizontal plane. It is indeed the Voronoï tessellation in the horizontal plane that should clearly show a signature of clustering as it is this plane that is perpendicular to gravity. An example of a Voronoï tessellation is included in Fig. 2(b). We take particles over the entire measurement height (roughly $550 \mathrm{~mm}$ ) so as to have as many samples as possible. Note that the choice of the vertical extent needs to be at least several times the typical vertical distance between descending particles in the same column, such that vertically aligned particles create dense regions once projected on the horizontal plane. We have checked that the PDF of the Voronoï areas is similar if a limited vertical extent (down to $125 \mathrm{~mm}$ ) is chosen. Each particle is assigned the set of all points (forming a convex polygon called a Voronoi cell) that is closest to itself rather than any other particle. The area of the cell is now inversely proportional to the local density [22]. Mathematically, the Voronoi tessellation is the dual graph of the Delaunay triangulation. We make sure not to include Voronoï cells at the periphery, which create artificially large cells, skewing the statistics.

The Voronoï tessellation is calculated for each frame and the area of the cells $\left(A_{\mathrm{V}}\right)$ is scaled by the mean area of each frame. The PDF of the normalized area $\left(A_{n}=A_{V} /\left\langle A_{V}\right\rangle\right)$ is calculated for each Ga; see Fig. 5. The figures include the corresponding PDF for random Poisson particles (RPP). For the lowest value of the Galileo number, $\mathrm{Ga}=110$, we already observe an increase in probability density for smaller and larger cells indicating some clustering, as we also observe in the angle-pair distribution in Fig. 4. For large Ga, we find even more increase in the probability 

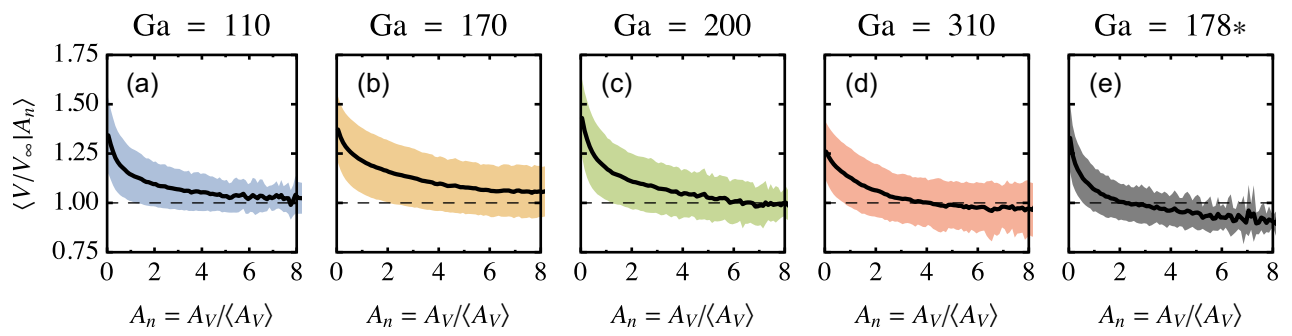

FIG. 6. Velocity of a particle conditioned on its normalized Voronoï area in the horizontal plane. Bands represent $\pm \sigma\left(v \mid A_{n}\right)_{A_{n}}$. Velocities are normalized using $V_{\infty}$, the terminal velocity of an isolated particle settling in a quiescent fluid. (e) Numerical data from Ref. [18]. Note that the "spiky" data for high $A_{n}$ are caused by a lack of statistics, and that the experiments have more statistics than the very expensive numerical simulations.

density for both small and large Voronoï areas, indicating a more pronounced clustering. Such an important level of clustering could already be observed from a simple visual inspection of Fig. 2(b): more dense and "open" areas than one would expect from randomly placed particles. Broadening of the distribution is also quantified by calculating the standard deviation of the distribution; see Fig. 5. Compared to an RPP $\left(\sqrt{\mu_{2}}=0.53\right)$, we indeed observe an increased standard deviation for $\mathrm{Ga}=110\left(\sqrt{\mu_{2}}=0.89\right)$, indicating clustering, and further increased standard deviations for even higher $\mathrm{Ga} \geqslant 170$ (up to $\sqrt{\mu_{2}}=1.25$ ). Increased standard deviation with respect to an RPP is indeed also what one observes in Fig. 2(b). We emphasize that the particles follow an RPP distribution when they exit the injector-very different from the distribution farther downstream.

A visual inspection of Fig. 2(b) also suggests that higher velocities are attained at high-density regions. To substantiate that claim the conditional average of the average settling velocity is calculated; see Fig. 6. The average velocity is conditioned on the normalized Voronoï area $A_{n}$ which is proportional to the inverse local density. Moreover, along with the mean velocity also the standard deviation is calculated for each $A_{n}$. The mean velocities and their spread are normalized using the terminal velocity of an isolated particle settling in a quiescent fluid bath. A clear increase in speed of up to $40 \%$ can be seen for high-density (low $A_{n}$ ) regions for all $\mathrm{Ga}$, and the graphs are very similar, showing the same behavior. As we stated before, the simulations of Refs. $[9,18]$ are performed in a three-dimensional periodic box where upward flow will certainly affect the velocity of some isolated particles. We can now clearly see this in Fig. 6(e); isolated particles $\left(A_{n} \gg 1\right)$ have normalized velocities below 1, meaning they settle slower than they would if they were to settle by themselves in an infinite bath. Also here we see that the case of $\mathrm{Ga}=310$ shows slightly less enhanced velocity (for example, at $A_{n}=0$ ) as compared to the $\mathrm{Ga}=170$ and $\mathrm{Ga}=200$ cases.

\section{CONCLUSION}

Starting from the visual observation in Fig. 2(a) that particles look vertically aligned, we find that, indeed, particles are preferentially aligned vertically as per our findings of the PDF of $\theta$ in Fig. 4. In the two-dimensional top view visual inspection suggests low- and high-density regions with low and high velocities, respectively. We confirm this view in Figs. 5 and 6. Altogether, our findings indicate that particles tend to settle in a preferential columnar configuration, with an increased trend to alignment with increasing Galileo number. This columnar alignment then impacts the settling of the particles, as particles that follow in the wake of another particle tend to settle faster. It was found in previous numerical simulations at $\mathrm{Ga}=121$ and $\mathrm{Ga}=178$ (although at a higher seeding density $\Phi_{V}=5 \times 10^{-3}$ ) by Uhlmann and Doychev [9] that only the latter tended to form columns impacting the settling. The different behavior between the low and high Galileo cases is interpreted in terms of the properties of particle wakes. The wakes of the low Ga particles $(\mathrm{Ga} \leqslant 155)$ have a stable vertical wake (see Fig. 1) and particles fall straight down. Only when two particles are already vertically aligned will the trailing particle fall in the wake of the leading particle. For higher Ga the 


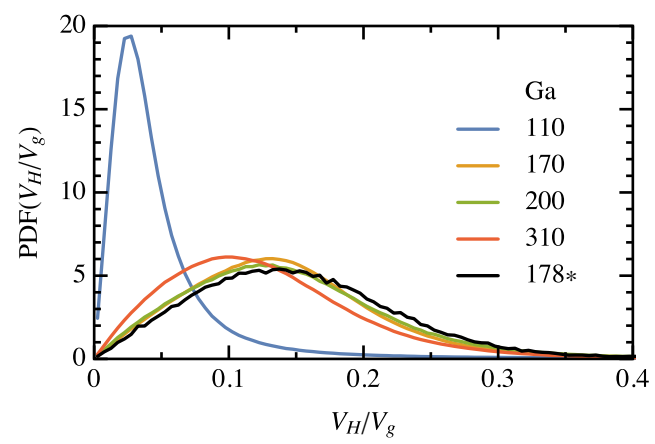

FIG. 7. PDF of the magnitude of the horizontal velocity $\left(V_{H}=\sqrt{V_{x}^{2}+V_{y}^{2}}\right)$ normalized by the gravitational velocity. Multiple experiments for the same Ga are in agreement and are combined to improve the quality of the statistics. Starred data are from numerical simulations and have $\Gamma=1.5[9,18]$, while the experiments have $\Gamma=2.5$.

wakes of the particles are unstable, causing the trajectory of said particles to be either steady oblique $(\mathrm{Ga} \geqslant 155)$, oblique oscillating $(\mathrm{Ga} \geqslant 185)$, or even chaotic $(\mathrm{Ga} \geqslant 215)$. In the aforementioned simulations, the case $\mathrm{Ga}=178$ was in a steady oblique regime; particles therefore had a horizontal motion with a higher chance of "catching" the slipstream of an upstream particle [9], causing the columnar alignment and the enhanced settling velocity. Our experimental results show that the same behavior (columnar alignment and enhanced settling) prevails for the oblique oscillating $(\mathrm{Ga}=200)$ and chaotic $(\mathrm{Ga}=310)$ situations which all exhibit lateral motion. The strongest impact on overall settling velocity enhancement is observed for particles at intermediate Galileo number $(\mathrm{Ga}=170)$ in the steady oblique situation. The highest Galileo case explored $(\mathrm{Ga}=310)$ was found on the contrary to exhibit less enhanced settling. At first glance this may seem consistent with the slightly reduced alignment observed for the $\mathrm{Ga}=310$ case from the angular statistics shown in Fig. 4. However, Voronoï statistics in Fig. 5 seem on the other hand to exhibit a slightly enhanced clustering for this same case, which would be on the contrary associated to settling enhancement. A first attempt of interpretation could be related to the chaotic nature of the particle motion at $\mathrm{Ga}=310$ which could still promote the chance for particles to catch each other's wake and form columns, while disturbing the alignment within the columns. However, the statistics of the horizontal velocity of the particles reveals a more complex situation. The PDF of the amplitude of the horizontal velocity $V_{H}=\sqrt{V_{x}^{2}+V_{y}^{2}}$ of the particles for $\mathrm{Ga}=310$ is indeed only marginally different as compared to the $\mathrm{Ga}=170, \mathrm{Ga}=178$, and $\mathrm{Ga}=200$ cases, but very different from the $\mathrm{Ga}=110$ case; see Fig. 7 . The $\mathrm{Ga}=110$ case exhibits a low (mean) sideways velocity compared to the other cases, with a narrow distribution, as expected for particles settling mostly in a "steady-straight" regime. The other cases exhibit all very similar PDFs compared to each other, with larger mean sideways velocities and broader distributions (note, though, that the mean sideways horizontal velocity is a little less for the $\mathrm{Ga}=310$ case as compared to the $\mathrm{Ga}=170, \mathrm{Ga}=178$, and $\mathrm{Ga}=200$ cases). Purely oblique trajectories (as expected for individual particles settling at $\mathrm{Ga}=170$ ) would exhibit a larger mean sideways motion compared to $\mathrm{Ga}=110$, but still a narrow PDF (the amplitude of the horizontal velocity being mostly constant). The chaotic case on the contrary is expected to have a wide PDF for the horizontal velocity which undergoes erratic fluctuations. The similarity of the PDFs for the four cases $\mathrm{Ga}=\{170,178,200,310\}$ therefore indicates that, though these particles follow different paths (Fig. 1) when they settle individually, an ensemble of particles eventually shows complex trajectories and chaotic wakes, probably due to particles and the wakes interacting with each other, causing horizontal velocities of the particles to have important and similar fluctuations (see Fig. 7). Furthermore, note that Fig. 7 shows the horizontal velocity of the particles and that the horizontal velocities of the flow in the wake of individual particles can follow a different trend. Overall these 
observations show that it is very likely irrelevant to speculate on possible interpretations for the differences in the settling collective behavior for particles at $\mathrm{Ga}=170$ and at $\mathrm{Ga}=310$ simply considering the usual "single particle regimes" in Fig. 1, although these regimes certainly play a role in the initial triggering of column formation. Why the $\mathrm{Ga}=310$ case shows less enhanced velocity, while still having a comparable $\operatorname{PDF}(\theta), \operatorname{PDF}\left(A_{n}\right),\left\langle V / V_{\infty} \mid A_{n}\right\rangle$, and $\operatorname{PDF}\left(V_{H} / V_{g}\right)$ (though in each case it slightly differs from the $\mathrm{Ga}=170, \mathrm{Ga}=178$, and $\mathrm{Ga}=200$ cases), is still an open question. Further research, where also the fluid velocity is measured, might help to find an explanation.

An increase or decrease in settling velocity is significant in cases where one wants to predict or prevent settling particles in e.g., chemical reactors with solid reactants or settling of particulate matter in riverbeds, and can probably also affect rainfall (the transition between steady vertical and steady oblique regimes for water droplets in air occurs for droplet diameters of the order of $850 \mu \mathrm{m}$ ) and ash cloud dynamics.

Finally, a striking observation of the present study is that, contrary to the simulations, we do find in the experiment that particles in the steady vertical regime $(\mathrm{Ga}=110)$ also exhibit mild clustering and column formation, although less pronounced than for particles at larger Galileo number (as shown by Voronoï statistics in Fig. 5 and the angular pair statistics in Fig. 4). A possible reason for the emergence of columns for such a low Galileo number, where no wake instability is expected, can be related to the existence of a large-scale flow caused by an ensemble of settling particles in a closed container such as ours. Such a large-scale flow is absent in the simulations presented in the paper as it has a periodic domain. This large-scale flow might induce some different flow dynamics as particles in low-density regions are less affected by upward flow as compared to those particles for the case of the simulation.

To summarize, we find a coherent set of observations for settling particles that explain the observed features: trajectory properties, vertical alignment, high-density regions, and enhanced settling velocity. Future studies will explore further the role of increasing the seeding density as well as the importance of confinement and boundary conditions. Another important extension of the present study concerns the impact of surrounding turbulence on the column formation and settling enhancement.

\section{ACKNOWLEDGMENTS}

We acknowledge the German-French program PROCOPE (Grant No. 57129319), the French program “TEC2" (Grant No. ANR-12-BS09-0011), and the German Research Foundation (DFG) under Project No. UH242/1-2 for funding this study.

[1] I. Newton, The Principia: Mathematical Principles of Natural Philosophy (University of California Press, 1999).

[2] T. A. Johnson and V. C. Patel, Flow past a sphere up to a Reynolds number of 300, J. Fluid Mech. 378, 19 (1999).

[3] B. Ghidersa and J. Dušek, Breaking of axisymmetry and onset of unsteadiness in the wake of a sphere, J. Fluid Mech. 423, 33 (2000).

[4] L. Schouveiler and M. Provansal, Self-sustained oscillations in the wake of a sphere, Phys. Fluids 14, 3846 (2002).

[5] G. Bouchet, M. Mebarek, and J. Dušek, Hydrodynamic forces acting on a rigid fixed sphere in early transitional regimes, Eur. J. Mech. B 25, 321 (2006).

[6] M. Jenny, J. Dušek, and G. Bouchet, Instabilities and transition of a sphere falling or ascending freely in a Newtonian fluid, J. Fluid Mech. 508, 201 (2004). 
[7] C. H. J. Veldhuis and A. Biesheuvel, An experimental study of the regimes of motion of spheres falling or ascending freely in a Newtonian fluid, Int. J. Multiphase Flow 33, 1074 (2007).

[8] M. Horowitz and C. H. K. Williamson, The effect of Reynolds number on the dynamics and wakes of freely rising and falling spheres, J. Fluid Mech. 651, 251 (2010).

[9] M. Uhlmann and T. Doychev, Sedimentation of a dilute suspension of rigid spheres at intermediate Galileo numbers: The effect of clustering upon the particle motion, J. Fluid Mech. 752, 310 (2014).

[10] P. Ern, F. Risso, D. Fabre, and J. Magnaudet, Wake-induced oscillatory paths of bodies freely rising or falling in fluids, Annu. Rev. Fluid Mech. 44, 97 (2012).

[11] J. F. Brady and G. Bossis, Stokesian dynamics, Annu. Rev. Fluid Mech. 20, 111 (1988).

[12] S. Ramaswamy, Issues in the statistical mechanics of steady sedimentation, Adv. Phys. 50, 297 (2001).

[13] É. Guazzelli and J. F. Morris, A Physical Introduction to Suspension Dynamics (Cambridge University Press, Cambridge, UK, 2011).

[14] R. N. Parthasarathy and G. M. Faeth, Turbulence modulation in homogeneous dilute particle-laden flows, J. Fluid Mech. 220, 485 (1990).

[15] M. Mizukami, R. N. Parthasarathy, and G. M. Faeth, Particle-generated turbulence in homogeneous dilute dispersed flows, Int. J. Multiphase Flow 18, 397 (1992).

[16] M. Uhlmann, An immersed boundary method with direct forcing for the simulation of particulate flows, J. Comput. Phys. 209, 448 (2005).

[17] T. Kajishima and S. Takiguchi, Interaction between particle clusters and particle-induced turbulence, Int. J. Heat Fluid Flow 23, 639 (2002).

[18] T. Doychev, The dynamics of finite-size settling particles, Ph.D. thesis, Karlsruhe Institute of Technology, 2014.

[19] R. Glowinski, T.-W. Pan, T. I. Hesla, and D. D. Joseph, A distributed Lagrange multiplier/fictitious domain method for particulate flows, Int. J. Multiphase Flow 25, 755 (1999).

[20] N. Machicoane, M. Lopez-Caballero, M. Bourgoin, A. Aliseda, and R. Volk, Improvements on particle tracking velocimetry: Model-free calibration and noiseless measurement of second order statistics of the velocity field, arXiv: 1605.03803.

[21] S. G. Huisman, P. Ern, and V. Roig, Interaction and coalescence of large bubbles rising in a thin gap, Phys. Rev. E 85, 027302 (2012).

[22] R. Monchaux, M. Bourgoin, and A. Cartellier, Preferential concentration of heavy particles: A Voronoi analysis, Phys. Fluids 22, 103304 (2010). 